\title{
Effects of rodent-induced disturbance on eco-physiological traits of Haloxylon ammodendron in the Gurbantunggut Desert, Xinjiang, China
}

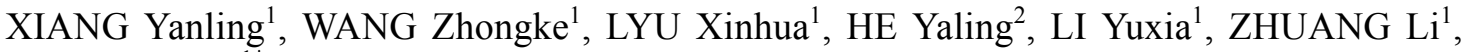 \\ ZHAO Wenqin ${ }^{1 *}$ \\ ${ }^{1}$ College of Life Sciences, Shihezi University, Shihezi 832003, China; \\ ${ }^{2}$ School of Medicine, Shihezi University, Shihezi 832003, China
}

\begin{abstract}
Disturbance by rodents alters the morphologies and nutrients of plants as well as the physical-chemical properties of the soils. Changes in plants are considered to be mechanisms of defense against the disturbance by rodents. Rodents gnaw on the assimilating branches of Haloxylon ammodendron (CA Mey.) Bunge and burrow under the bushes in the desert ecosystems of Xinjiang, China. However, eco-physiological responses of different age groups of $H$. ammodendron to the disturbance by rodents are not well understood. In this study, soil physical-chemical properties under the shrubs and the above-ground morphological, physiological and biochemical features of assimilating branches of $H$. ammodendron of different age groups (i.e., young, 30-100 cm; middle-aged, 100-200 cm; and mature, $>200$ $\mathrm{cm}$ ) in burrowed and non-burrowed (control) areas were studied in 2018. We found that disturbance by rodents significantly increased the crown width and total branching rates of young and middle-aged $H$. ammodendron. Photosynthetic pigment contents of assimilating branches of $H$. ammodendron were significantly reduced under the disturbance by rodents. In term of plant nutrients, the main differences among different age groups of $H$. ammodendron under the disturbance by rodents occurred in the total soluble sugar and reducing sugar contents that decreased in young plants, increased in middle-aged plants, and did not affect in mature plants. Crude protein and phosphorus contents significantly increased, while crude fiber and calcium contents significantly decreased in young plants. Crude fat and calcium contents significantly decreased in middle-aged plants. Soil organic matter ( $\mathrm{SOM}$ ), total nitrogen (TN), available nitrogen (AN) and available potassium (AK) contents in the topsoil $(0-20 \mathrm{~cm})$, which are conducive to forming "fertile islands", also increased under the disturbance by rodents. In particular, soil AN and AK were the major factors affecting the above-ground morphological characteristics of $H$. ammodendron in burrowed areas. Overall, the response and defense strategies of $H$. ammodendron to the disturbance by rodents differed among different age groups, and the effect of the disturbance by rodents on $H$. ammodendron gradually weakened with the increasing plant age.
\end{abstract}

Keywords: age groups; morphology; assimilating branches; soil physical-chemical properties; photosynthetic pigments

Citation: XIANG Yanling, WANG Zhongke, LYU Xinhua, HE Yaling, LI Yuxia, ZHUANG Li, ZHAO Wenqin. 2020. Effects of rodent-induced disturbance on eco-physiological traits of Haloxylon ammodendron in the Gurbantunggut Desert, Xinjiang, China. Journal of Arid Land, 12(3): 508-521. https://doi.org/10.1007/s40333-020-0015-8

\footnotetext{
*Corresponding author: ZHAO Wenqin (E-mail: zhwq-88@163.com)

Received 2019-06-01; revised 2020-02-05; accepted 2020-03-25

(C) Xinjiang Institute of Ecology and Geography, Chinese Academy of Sciences, Science Press and Springer-Verlag GmbH Germany, part of Springer Nature 2020
} 


\section{Introduction}

Haloxylon ammodendron (CA Mey.) Bunge, a representative $\mathrm{C}_{4}$ succulent xerophyte grows in the desert areas of northwestern China, has xeromorphic characteristics for adaptation to drought and high-salinity environments. Recently, researchers found that $H$. ammodendron provides a high nutrient content to herbivorous animals in the desert environments (Kang et al., 2017). The assimilating branches of $H$. ammodendron provide not only nutrients but also water for rodents living in the desert environments (Mares et al., 1997). Rodents tend to cut the branches horizontally into small pieces and carry them to their burrows for food (Mabry et al., 1977). Additionally, fossorial mammals construct burrow systems where food, feces, seeds, plant propagules and numerous types of organic material are stored, creating patches rich in organic content and affecting the dispersion of soil nutrients (Lacey et al., 2001). Therefore, the life activities of rodents are certain to have a profound impact on the growth of $H$. ammodendron.

Growth differentiation balance hypothesis projects that plants could progress into a "growth or defense" stage, either by investing resources in defenses against herbivores or by out-growing competitors (Siemens et al., 2002). Animal feeding damage might change the morphology of plants because of the effects of selective feeding on particular plant tissues (Andino and Borghi, 2018). In certain cases, phenotypic plasticity is regarded as a low-cost defense mechanism that has a crucial role in plant-herbivore interactions (Hakes and Cronin, 2011; Kozlov and Zvereva, 2012; Dong et al., 2017). When the shoot apices of plants have been detached by herbivores, damaged plants have been documented to generate axillary branches to improve photosynthesis and compensate for tissue damage (Christie et al., 2014; Dong et al., 2017). A study by Zhang et al. (2009) also showed that slight damage to $H$. ammodendron by foraging gerbils promoted an increase in the growth of the branches and crown. Nutritional grade of a plant is reflected in its probable resistance factor once the nutrient content is outside the optimal array of herbivores (Kindler and Robert, 1970; Schweissing and Wilde, 1979). A deficiency in plant nutrients could decrease plant palatability (Ngatia et al., 2015), making plants less desirable to herbivores. Furthermore, the effects of herbivores on plants might differ among different plant growth stages. Studies have shown that disturbance of herbivores increased the defense compounds (e.g., alkaloids) of offspring plants (Barton and Koricheva, 2010; Kafle and Wurst, 2019). The increase of defense compounds may reduce the investment in growth or the synthesis of nutrients (Züst et al., 2015; Karasov et al., 2017). However, eco-physiological responses of different age groups of $H$. ammodendron to the disturbance by rodents remain unclear. In this paper, morphological characteristics of above-ground parts and the level of nutrients in assimilating branches of $H$. ammodendron of different age groups, which is located at the southern edge of the Gurbantunggut Desert, under the disturbance of rodents were researched.

Rodents can modify the physical-chemical properties of soil via their burrowing activities (Berke, 2010; Hagenah and Bennett, 2013). Recent studies have shown that the activities of burrowing animals play an important role in promoting the formation of "fertile islands", which usually manifests as the increases in soil nitrogen, phosphorus and carbon contents (Xu et al., 2012; Allington and Valone, 2014). Thus, the disturbance by rodents may be beneficial to the growth of $H$. ammodendron. Therefore, we tested the physical-chemical properties of below-shrub soil at various depths in burrowed and control areas. Furthermore, the contents of leaf photosynthetic pigments are valuable indicators for possible photosynthetic efficiency (Mao et al., 2007). Therefore, we also compared the differences in the photosynthetic pigment contents of assimilating branches of $\mathrm{H}$. ammodendron of different age groups in burrowed and control areas. We hypothesized that: (1) disturbance by rodents would lead to a reduction in photosynthetic pigments of assimilating branches of $\mathrm{H}$. ammodendron. To compensate for nutrient and photosynthetic losses caused by the disturbance, $H$. ammodendron might alter its morphology; (2) $H$. ammodendron would weaken rodent feeding on assimilating branches by reducing their nutrient contents. However, changes in nutrient contents in assimilating branches of $H$. ammodendron may differ among different age groups; (3) rodent activities would increase the contents of organic matter, nitrogen, phosphorus and potassium in the soil under H. ammodendron 
shrubs, which is likely to stimulate the growth of $H$. ammodendron to some degree; and (4) responses of $H$. ammodendron to the disturbance by rodents would differ among different age groups. In addition, eco-physiological responses of young $H$. ammodendron to the disturbance by rodents would be more dramatic than those of middle-aged and mature $H$. ammodendron.

\section{Materials and methods}

\subsection{Study area}

Study area is located at the southern edge of the Gurbantunggut Desert, at a transitional zone between desert and oasis $\left(45^{\circ} 02^{\prime} \mathrm{N}, 85^{\circ} 04^{\prime} \mathrm{E} ; 346 \mathrm{~m}\right.$ a.s.1.). The study area belongs to a typical temperate continental climate with the mean annual precipitation of $117 \mathrm{~mm}$. The mean annual evaporation is more than $1980 \mathrm{~mm}$ and annual mean temperature is $6.6^{\circ} \mathrm{C}$. The maximum temperature can reach $43.1^{\circ} \mathrm{C}$ in summer and the minimum temperature $-27.0^{\circ} \mathrm{C}$ in winter. The surface of the landscape is a mosaic of dry riverbed and aeolian sandy landform. In this area, xerophytic plants are the most common type of vegetation. The vegetation is dominated by $H$. ammodendron and Haloxylon persicum with approximately 30\% coverage (Huang et al., 2015), where $H$. ammodendron is dispersed between the dunes. Rhombomys opimus, Cricetulus migratorius, Meriones meridianus and Dipus sagitta are typical rodent species in this area (Xu et al., 2012). Amongst them, Rhombomys opimus is the most abundant species. Ma et al. (2018) found that the coverage of burrows in the southern edge of the Gurbantunggut Desert in Xinjiang was in the range of $18 \%-20 \%$.

\subsection{Experimental design}

In July 2018, we searched for rodent-burrowed areas along the direction of a longitudinal dune. Three $10 \mathrm{~m} \times 10 \mathrm{~m}$ quadrats were placed in the middle of burrowed area. Taking the burrowed area as a center, we set up three $10 \mathrm{~m} \times 10 \mathrm{~m}$ control quadrats in areas distinct from the edge of burrowed area, where there was no (or limited) signs of burrowing, no fresh rodents feces on the ground or at the burrowed entrances and no recently plowed sand at the burrowed entrances (Tchabovsky et al., 2001). The distances between all quadrats were over $50 \mathrm{~m}$. Two test replicates were set at a distance of more than $1 \mathrm{~km}$ from the test site, covering three burrowed areas in total (control areas).

\subsection{Measurement of morphological characteristics of above-ground plant parts}

Plant height, basal stem diameter, crown width and total branching rate in the quadrats were measured for all $H$. ammodendron. Basal stem diameter was measured approximately $3 \mathrm{~cm}$ above the ground. Crown width was expressed as the average width of $H$. ammodendron in the east-western and north-southern directions. Total branching rate $\left(R_{\mathrm{b}}\right)$ of $H$. ammodendron was calculated using the formula of Whitney (1976) and Steingraeber and Waller (1986).

$$
R_{\mathrm{b}}=(\mathrm{NT}-\mathrm{NS}) /(\mathrm{NT}-\mathrm{N} 1) \text {, }
$$

where NT is the total number of branches; NS is the highest branch number; and N1 is the first branch number.

\subsection{Collection and analysis of assimilating branches}

Five plants from each young, middle-aged and mature $H$. ammodendron were carefully chosen. A total of fifteen plants were selected from burrowed and control areas. H. ammodendron in the height range of 30-100 cm was considered as young plant, $H$. ammodendron of 100-200 cm was considered as middle-aged plant, and those over $200 \mathrm{~cm}$ was considered as mature plant (Zhang et al., 2016; Luo et al., 2017). Assimilating branches of $H$. ammodendron in all four directions of east, south, west and north were collected (annual branches were used to analyze photosynthetic pigments, and 1 a to 3 a branches were used to analyze nutrient contents). Assimilating branch samples were fully mixed within different areas and age groups, then numbered and brought back to the laboratory. We extracted chlorophylls (total, i.e., $a$ and $b$ ) and carotenoids with $80 \%$ acetone and determined their concentrations spectro-photometrically according to the method of 
Lichtenthaler (1987). Assimilating branch samples were dried in an oven at $65^{\circ} \mathrm{C}$ until a constant weight was achieved, and then crushed by grinder. After grinding, the samples were passed through the 40-mesh sieves for further measurement of nutrient content. According to the methods of Roe (1955) and Nelson (1944), we quantified total soluble sugar and reduced sugar with glucose as the standard. Crude protein content was measured by the Kjeldahl method, and the conversion coefficient was 6.25. Crude fat content was determined with diethyl-ether extract by the Soxhlet method. Crude fiber content was measured by the weight method (Somogyi, 1952). Phosphorous and calcium contents were measured using atomic absorption spectrum-photometry (AAS, Type 932GBC, Scientific Equipment Pty Ltd., Australia).

\subsection{Soil collection and analyses}

We carefully selected an area of ground under the canopy cover of fifteen plants each of young, middle-aged, and mature $H$. ammodendron in burrowed and control areas. For each chosen area, soil samples were collected at the four compass directions and three depths $(0-20,20-40$ and $40-60 \mathrm{~cm}$ ) at about $20 \mathrm{~cm}$ from the base trunk of the shrub, and samples were mixed to form a single composite sample. The soil samples were sealed, labelled and brought back to the laboratory for analysis. All soil samples were dried naturally, ground and passed through a $0.2-\mathrm{mm}$ sieve for analysis. Soil $\mathrm{pH}$ value (1:5 soil:water mix) was measured using a $\mathrm{pH}$ meter (PHS-25 digital explicit, Shanghai Yi Dian Appliance Co., Ltd., China). The following soil properties were measured using the methods of Bao (1999): soil organic matter content (SOM, $\mathrm{KCr}_{2} \mathrm{O}_{7}$ method), total nitrogen ( $\mathrm{TN}, \mathrm{HClO}_{4}-\mathrm{H}_{2} \mathrm{SO}_{4}$ digestion method), total phosphorus (TP, $\mathrm{Mo}-\mathrm{Sb}$ colorimetric method), total potassium (TK, atomic absorption spectrometry), available nitrogen ( $\mathrm{AN}, \mathrm{CaCl}_{2}$ extracts analyzed with a flow analyzer), available phosphorus (AP, $\mathrm{NaHCO}_{3}$ extracts analyzed with the Mo-Sb colorimetric method) and available potassium (AK, $\mathrm{NH}_{4} \mathrm{OAc}$ extracts analyzed by atomic absorption spectrometry).

\subsection{Data analyses}

Softwares of SPSS 19.0 and Origin 9.5 are used for data analysis and plotting. The data in the figures are represented by means $\pm \mathrm{SE}$. The above-ground morphology of $H$. ammodendron, physiological characteristics of assimilating branches, and soil physical-chemical properties in burrowed and control areas were compared using an independent-sample $T$-test method $(P<0.05)$. The concentrations of photosynthetic pigments in assimilating branches of $H$. ammodendron of different ages and the physical-chemical properties of the soil at different depths were compared by means of a one-way ANOVA (analysis of variance) test and LSD (least significant difference) multiple comparisons. Pearson's correlation analysis was used to analyze the correlations among the above-ground morphological characteristics. Canoco 5.0 redundancy analysis (RDA) was used to examine the associations among plant above-ground morphological characteristics and topsoil $(0-20 \mathrm{~cm})$ physical-chemical properties in burrowed and control areas $(P<0.05)$. Before the analysis, above-ground morphological characteristics and soil physical-chemical properties were converted into $\lg (x+1)$ form. Detrended correspondence analysis (DCA) was used to analyze the above-ground morphological characteristics (Lepš and Šmilauer, 2003). The outcomes showed that the extended gradients at all axes in burrowed and control areas were 0.325 and 0.328 , respectively. As these were less than 3.000, they were suitable for linear redundancy analysis. All the expansive factors of soil physical-chemical properties were less than 10.000; therefore, they were all analyzed by RDA (Braak and Prentice, 1988). Monte Carlo Permutation tests were used to progressively choose significant soil physical-chemical properties.

\section{Results}

\subsection{Effects of disturbance by rodents on above-ground morphological characteristics of $\boldsymbol{H}$. ammodendron}

Disturbance by rodents reduced the mean height of $H$. ammodendron, but the difference was not significant among different age groups $(P<0.05$; Fig. 1a). The effect of disturbance by rodents on 
the above-ground morphological characteristics was more pronounced for young $H$. ammodendron. The base diameter and crown width of young $H$. ammodendron in burrowed areas were significantly larger than those in control areas $(P<0.05)$ by $38.92 \%$ and $41.43 \%$, respectively (Fig. 1b). Disturbance by rodents significantly increased the crown width of middle-aged $H$. ammodendron by $21.17 \%(P<0.05$; Fig. 1c). Total branching rates of young, middle-aged and mature $H$. ammodendron significantly increased by $20.88 \%, 38.65 \%$ and $48.19 \%$, respectively $(P<0.05$; Fig. 1d) under the disturbance by rodents.

The height of $H$. ammodendron showed a close positive correlation with its basal stem diameter; nonetheless, this relationship was more significant in the control areas than in the burrowed areas $(P<0.01$; Fig. 1e). A close positive correlation was found between the height and crown width of H. ammodendron in control areas $(P<0.01$; Fig. 1f). However, low-level positive correlation was also found between the height and crown width of $H$. ammodendron in burrowed areas $(P<0.05$; Fig. 1f). It appears that the effect of disturbance by rodents on crown width was greater than that on basal stem diameter.
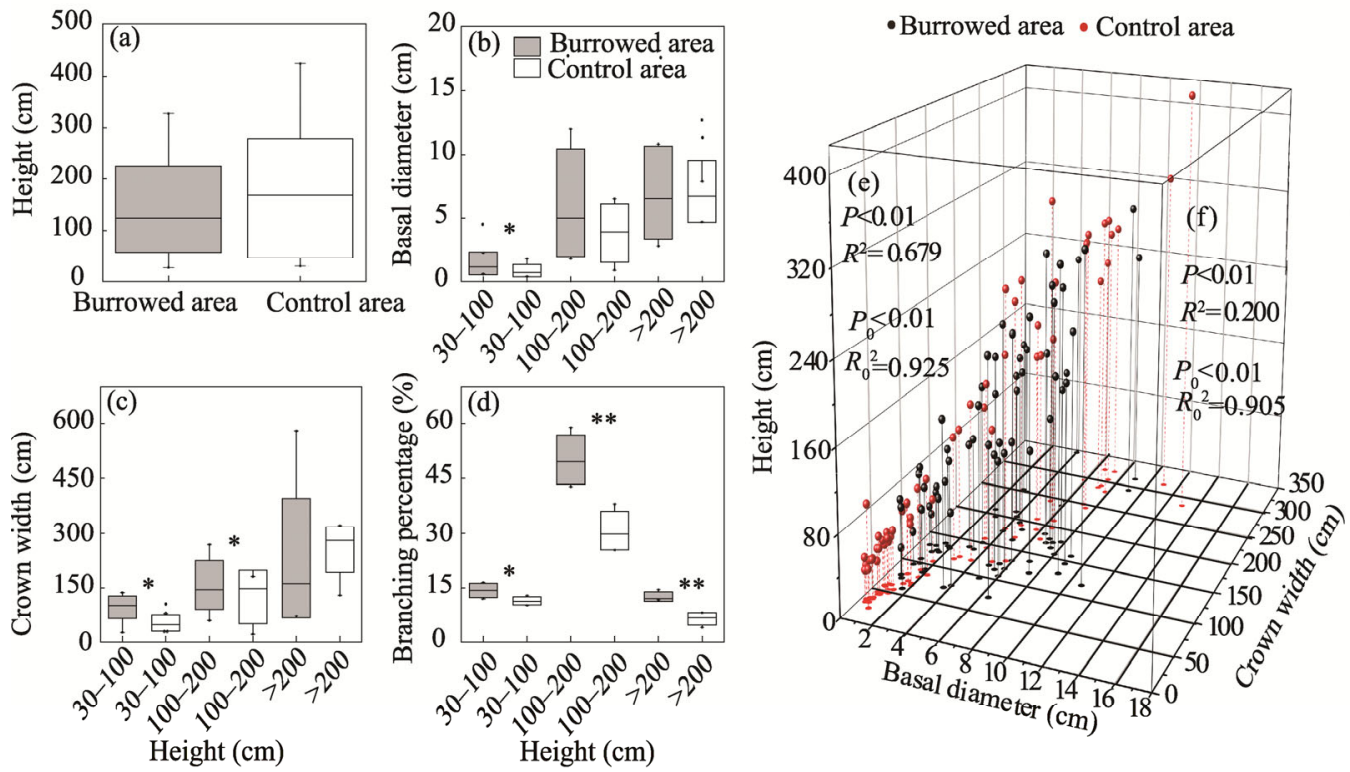

Fig. 1 Difference in above-ground morphological characteristic (a-d) of $H$. ammodendron with different ages (heights) in burrowed and control areas. Boxes represent the interquartile range (containing $50 \%$ of values), lines across boxes represent medians, whiskers indicate the highest and lowest values, and circle indicates outlier. ${ }^{*}$ and ** indicate significant differences between burrowed and control areas at $P<0.05$ and $P<0.01$ levels, respectively. (e), Correlation between height and basal stem of $H$. ammodendron in burrowed $\left(R^{2}\right)$ and control $\left(R_{0}^{2}\right)$ areas; and (f) correlation between height and crown width of $H$. ammodendron in burrowed $\left(R^{2}\right)$ and control $\left(R_{0}^{2}\right)$ areas.

\subsection{Effects of disturbance by rodents on physiological characteristics of assimilating branches}

3.2.1 Nutrient content in assimilating branches

Effects of disturbance by rodents on the nutrient content of assimilating branches of $H$. ammodendron differed among different age groups (Fig. 2). Disturbance significantly reduced the content of most nutrients, i.e., total soluble sugar, reducing sugar, crude fiber and calcium, in assimilating branches of young $H$. ammodendron, and they decreased by $15.21 \%, 25.78 \%$, $15.74 \%$, and $17.40 \%$, respectively. However, crude protein and phosphorus contents were $18.04 \%$ and $15.62 \%$ higher in borrowed areas than in control areas (Figs. 2a, c, d, e and f). In contrast, total soluble sugar and reducing sugar contents in assimilating branches of middle-aged $H$. ammodendron in burrowed areas were $29.44 \%$ and $27.05 \%$ greater than those of in control areas, whereas, crude fat and calcium contents were $16.36 \%$ and $33.01 \%$ lower in burrowed areas than in control areas (Figs. 2b, c, e and f). For mature H. ammodendron, disturbance by rodents only 
reduced the phosphorus content in assimilating branches $(P<0.05$; Fig. 2). Overall, the change in nutrient contents in assimilating branches was the lowest in mature H. ammodendron compared with young and middle-aged $H$. ammodendron.
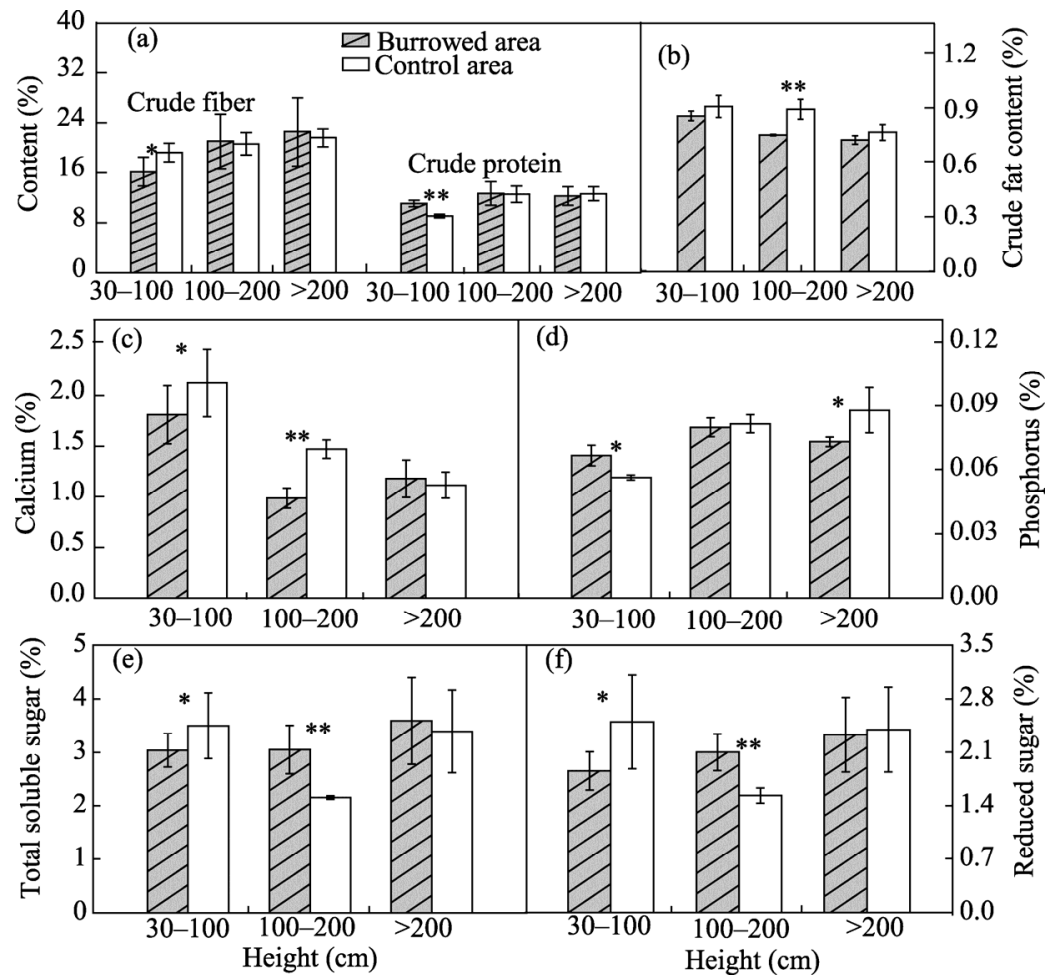

Fig. 2 Difference of nutrient content in assimilating branches of H. ammodendron in burrowed and control areas. Bars represent standard error. ${ }^{*}$ and $* *$ indicate significant differences between burrowed and control areas at $P<0.05$ and $P<0.01$ levels, respectively.

3.2.2 Photosynthetic pigment content in assimilating branches

Disturbance by rodents had a significant effect on the photosynthetic pigment content of assimilating branches of $H$. ammodendron. Photosynthetic pigment content of young $H$. ammodendron was significantly reduced $(P<0.05$; Fig. 3$)$. And in middle-aged $H$. ammodendron, photosynthetic pigment content was significantly decreased, but there was no significant effect on carotenoids $(P<0.01$; Fig. 3a). Chlorophyll $a$, total chlorophyll (Chlorophyll $a$ and $b$ ) and carotenoid contents of assimilating branches of mature $H$. ammodendron significantly decreased in burrowed areas $(P<0.05$; Fig. 3$)$.
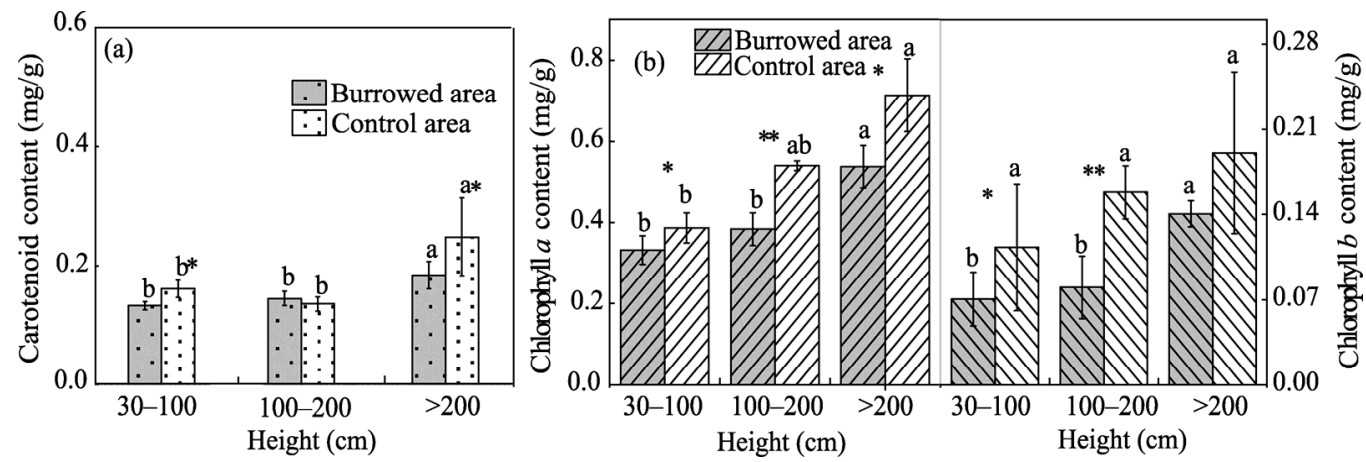

Fig. 3 Comparison of photosynthetic pigment content of $H$. ammodendron in burrowed and control areas. Different lowercase letters represent significant differences among different age groups (heights) of $H$. ammodendron at $P<0.05$ level. * and ** indicate significant differences between burrowed and control areas at $P<0.05$ and $P<0.01$ levels, respectively. 


\subsection{Soil physical-chemical property}

Soil depth had no significant effect on the contents of SOM, TP, AP, TK and AK in burrowed and control areas $(P>0.05$; Fig. 4). Soil TN content was the highest in the surface soil $(0-20 \mathrm{~cm})$. With increasing soil depth, soil TN content decreased by $55.56 \%$ and $25.00 \%$ in burrowed and control areas, respectively; whereas, soil water content increased by $75.60 \%$ and $67.75 \%$ in burrowed and control areas, respectively $(P<0.05$; Fig. 4$)$. Disturbance by rodents enhanced the "fertile island" effect of $H$. ammodendron, which mainly manifested as an increase in soil AN content at the depth of 0-40 cm, especially in the topsoil $(0-20 \mathrm{~cm})$. SOM, TN, AN and AP contents significantly increased by $20.09 \%, 55.57 \%, 45.06 \%$ and $8.94 \%$, respectively, at the $0-20$ $\mathrm{cm}$ depth in burrowed areas compared with control areas $(P<0.05$; Fig. 4$)$. AN and AP contents and $\mathrm{pH}$ of soil at the depth of $20-40 \mathrm{~cm}$ were significantly higher in burrowed areas than in control areas $(P<0.05$; Fig. 4), having increased by $40.39 \%, 22.71 \%$ and $1.57 \%$, respectively. However, soil $\mathrm{pH}$ at the depth of $40-60 \mathrm{~cm}$ in burrowed areas was significantly lower $(2.12 \%)$ than that in control areas $(P<0.01$; Fig. 4). In addition, disturbance by rodents significantly reduced the water content of surface soil $(0-20 \mathrm{~cm})$ by $35.56 \%(P<0.05$; Fig. 4$)$.
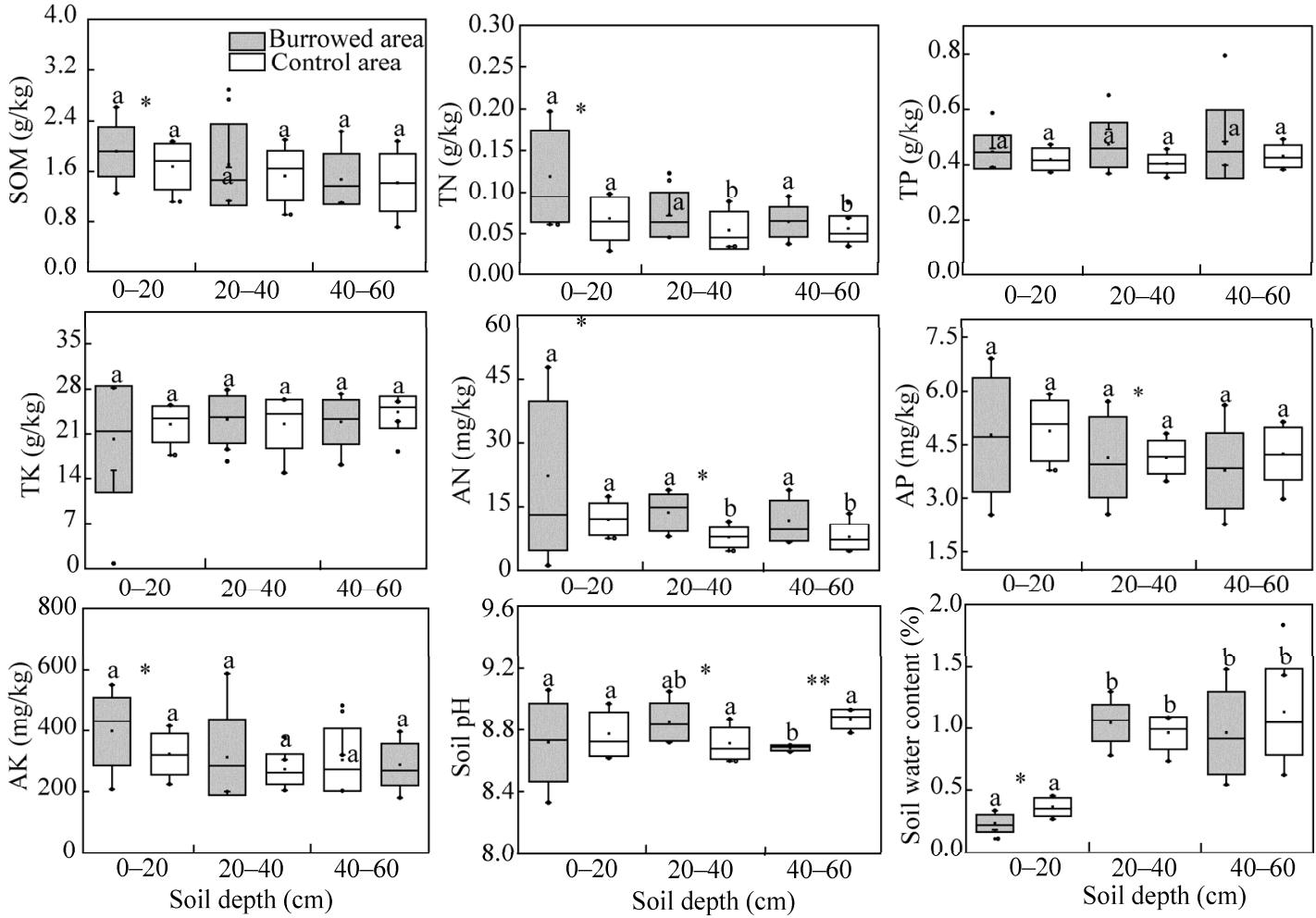

Fig. 4 Difference in soil physical-chemical property of $H$. ammodendron in burrowed and control areas. Different lowercase letters indicate significant difference among different soil depths at $P<0.05$ level. Boxes represent the interquartile range (containing $50 \%$ of values), lines across boxes represent medians, whiskers indicate the highest and lowest values, and circle indicates outlier. ${ }^{*}$ and $* *$ indicate significant differences between burrowed and control areas at $P<0.05$ and $P<0.01$ levels, respectively. SOM, soil organic matter; TN, total nitrogen; TP, total phosphorus; TK, total potassium; AN, available nitrogen; AP, available phosphorus; AK, available potassium.

\subsection{Correlation between soil physical-chemical property and above-ground morphological characteristic}

RDA was performed to evaluate the associations between physical-chemical properties of topsoil $(0-20 \mathrm{~cm})$ and the above-ground morphology of $H$. ammodendron (Fig. 5; Table 1). In this experiment, there was no significant difference in soil water content among the deep soil samples (data not shown). Hence, soil water content was not included in the RDA analysis. The first two 
axes elucidated $70.2 \%$ of the morphological characteristics of burrowed areas (Fig. 5a) and $63.8 \%$ of the morphological features of control areas (Fig. 5b). AN $(P=0.002)$ and AK $(P=0.002)$ had important influences on the above-ground morphology of $H$. ammodendron in burrowed areas and the order of influence was AN $>$ AK (Fig. 5a). In control areas, AN $(P=0.02), \mathrm{AK}(P=0.004)$, TN $(P=0.012)$, and AP $(P=0.008)$ had notable effects on the above-ground morphology of $H$. ammodendron, with the order of influence being $\mathrm{AN}>\mathrm{AK}>\mathrm{TN}>\mathrm{AP}$ (Fig. 5b). The results indicate that $\mathrm{AN}$ and $\mathrm{AK}$ in the topsoil are the most factors affecting the above-ground morphology of $H$. ammodendron in the presence or absence of disturbance by rodents.
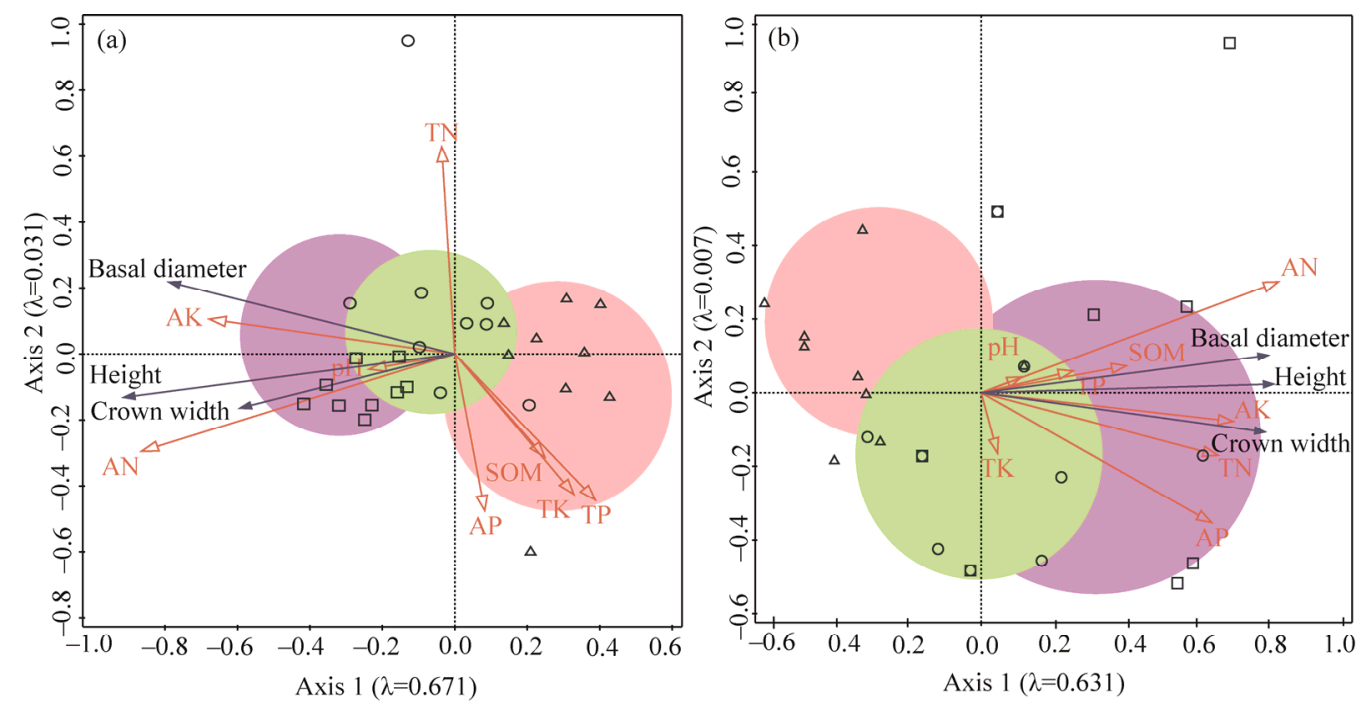

Fig. 5 Correlation between physical-chemical property of topsoil and above-ground morphology of $H$. ammodendron, as determined by redundancy analysis (RDA). (a), burrowed areas; and (b), control areas. $\Delta$, young $H$. ammodendron;, , middle-aged $H$. ammodendron; $\square$, mature $H$. ammodendron; SOM, soil organic matter; TN, total nitrogen; TP, total phosphorus; TK, total potassium; AN, available nitrogen; AP, available phosphorus; AK, available potassium.

Table 1 Forward selection of soil variables during the redundancy analysis

\begin{tabular}{|c|c|c|c|c|c|}
\hline Sample plot & Soil variable & Eigenvalues & Explained variance $(\%)$ & $F$ & $P$ \\
\hline \multirow[t]{8}{*}{ Burrowed area } & $\mathrm{AN}$ & 0.505 & 50.5 & 25.5 & 0.002 \\
\hline & $\mathrm{AK}$ & 0.309 & 30.9 & 11.2 & 0.002 \\
\hline & $\mathrm{TP}$ & 0.107 & 10.7 & 3.0 & 0.072 \\
\hline & TK & 0.078 & 7.8 & 2.1 & 0.122 \\
\hline & SOM & 0.045 & 4.5 & 1.2 & 0.270 \\
\hline & $\mathrm{pH}$ & 0.038 & 3.8 & 1.0 & 0.354 \\
\hline & AP & 0.018 & 1.8 & 0.5 & 0.578 \\
\hline & $\mathrm{TN}$ & 0.014 & 1.4 & 0.3 & 0.690 \\
\hline \multirow[t]{8}{*}{ Control area } & $\mathrm{AN}$ & 0.426 & 42.6 & 18.5 & 0.002 \\
\hline & $\mathrm{AK}$ & 0.305 & 30.5 & 11.0 & 0.004 \\
\hline & $\mathrm{TN}$ & 0.270 & 27.0 & 9.2 & 0.012 \\
\hline & AP & 0.256 & 25.6 & 8.6 & 0.008 \\
\hline & SOM & 0.101 & 10.1 & 2.8 & 0.104 \\
\hline & $\mathrm{TP}$ & 0.041 & 4.1 & 1.1 & 0.294 \\
\hline & $\mathrm{pH}$ & 0.008 & 0.8 & 0.2 & 0.762 \\
\hline & TK & 0.002 & 0.2 & $<0.1$ & 0.878 \\
\hline
\end{tabular}

Note: AN, available nitrogen; AK, available potassium; TP, total phosphorus; TK, total potassium; SOM, soil organic matter; AP, available phosphorus; $\mathrm{TN}$, total nitrogen. 


\section{Discussion}

\subsection{Soil physical-chemical property under the shrub of $\boldsymbol{H}$. ammodendron}

In this study, rodent activity significantly increased SOM, TN, AN and AP contents in the soil (Fig. 4). This is mainly due to the burrow system constructed by rodents under the shrub of $H$. ammodendron, resulting in an accumulation in plant residues, feces and urine in the soil and strengthening the "fertile island" effect. However, the difference was most notable in the topsoil $(0-20 \mathrm{~cm})$ (Fig. 4) because of the range of rodent activity. Studies have shown that gerbils, the most abundant rodent species in this area, are mainly active at the 20-30 cm soil depth (Jiang et al., 2007; $\mathrm{Xu}$ et al., 2012). In addition to their underground activities, the frequent surface activities of rodents also lead to an increase in nutrients in the topsoil. The increase in soil AN and AP contents at the 20-40 cm depth in burrowed areas may be related to the activity of microorganisms. Studies have shown that increase in organic matter and nitrogen contents of the soil increases microbial biomass and diversity, improving the bio-availability of minerals (Ewing et al., 2007; Jiang et al., 2007). In addition, the activities of rodents are likely to affect the release of exudates from the roots of $H$. ammodendron, thus affecting the physical-chemical properties of soil and the biomass and diversity of microorganisms. Moreover, this interaction may be more obvious in rhizosphere soil. However, this speculation needs further study.

As a typical salt-tolerant plant, the salt content of the soil under H. ammodendron is high, due to the absorption of water and transport of salt by the shrub's root system ( Munns and Tester, 2008; He et al., 2018), a phenomenon known as the "salt island" effect ( $\mathrm{Li}$ et al., 2011). In our study, interference by rodents significantly increased the soil pH at the depth of 20-40 cm (Fig. 4). The activities of rodents may have an impact on the "salt island" effect under $H$. ammodendron. Damage to the roots of $H$. ammodendron through the activities of rodents may weaken its ability to absorb and transport salt. Our study did not involve the determination of soil salt content, so further relevant research is needed. In addition, because the activities of rodents vary among different seasons (Liu et al., 2012), the physical-chemical changes in the soil of burrow areas will vary depending on the time of soil sample collection. For example, in the season when rodents store food, the number of foraging holes will vastly increase, which will affect the physical-chemical properties of the soil (Whitford and Kay, 1999). Studies into changes in the soil physical-chemical properties due to disturbance by rodents throughout the seasons are needed to resolve this question.

\subsection{Above-ground morphological characteristic of $\boldsymbol{H}$. ammodendron}

We found that AN and AK were positively correlated with the height, base diameter and crown width of $H$. ammodendron and were the main determining factors affecting the growth of $H$. ammodendron in areas disturbed by rodents (Fig. 5a). This might be because the return of nutrients to the soil is slow in desert ecosystems (Barthelemy et al., 2018), and $\mathrm{N}$ and $\mathrm{K}$ sources in the rodent feces could be readily absorbed by vascular plants to stimulate plant growth (Sitters et al., 2017). However, disturbance by rodents had a minor inhibitory effect on plant height, although the difference was not statistically significant (Fig. 1a). The results show that herbivores have a small inhibitory effect on the growth of $H$. ammodendron. Young $H$. ammodendron in burrowed areas had thicker stems than those in control areas (Fig. 1b), and this was probably related to the subsequent increase in soil nutrients. However, disturbance by rodents only affected the base diameter of young $H$. ammodendron. In addition, young $H$. ammodendron disturbed by rodents had greater crown widths and total branching rates than those of undisturbed plants (Figs. $1 \mathrm{c}$ and $\mathrm{d}$ ), which is likely to be related to the increases in the soil nutrient content and obtainability. The increased branching rate may also be triggered by amputation, which tends to lead to an increase in crown width. Amputation generally leads to the greater branch strength, which is induced by axillary meristems released from the apical dominance (Shimizu-Sato et al., 2009). Furthermore, effect of the disturbance by rodents on the total branching rate of middle-aged and mature $H$. ammodendron was much greater than that of young $H$. ammodendron 
(Fig. 1d), which may be due to the fact that rodents favor larger plants when building burrows, and they move short distances for small periods of time, staying near burrows most of the time (Falkenberg and Clarke, 1998; Rogovin et al., 2003). Hence, larger H. ammodendron display a greater branching response to damage. The increased crown width and branching help to increase the leaf area of $H$. ammodendron, allowing it to absorb more sunlight, $\mathrm{CO}_{2}$ and $\mathrm{O}_{2}$. To a degree, this is also because of the morphological flexibility of $H$. ammodendron in response to the disturbance by rodents.

\subsection{Photosynthetic pigment and nutrient contents of assimilating branches of $H$. ammodendron}

Disturbance by rodents significantly decreased the photosynthetic pigment content of annual assimilating branches of $H$. ammodendron, except for the carotenoid content in assimilating braches of middle-aged $H$. ammodendron (Fig. 3). It is well known that jasmonic acid (JA) or methyl jasmonate (JA-Me) promotes chlorophyll degradation in leaves (Jung, 2004; Li et al., 2016). Both mechanical injury and herbivore attack may lead to a rapid accumulation of JA or JA-Me in plants (Campos-Vargas and Saltveit, 2010; Nabity et al., 2013). Hence, we speculate the reduction in chlorophyll content triggered by disturbance by rodents might be associated with JA-induced chlorophyll degradation after assimilating branches were fed. Furthermore, mechanical damage to chloroplasts and reactive oxygen species-mediated lipid peroxidation of chlorophyll pigments may also be the influencing factors of chlorophyll content reduction (Sivritepe et al., 2009). However, this phenomenon requires further study.

Compared with control areas, there were no significant correlations among the content of photosynthetic pigments and the total soluble sugar or reducing sugar of assimilating branches in burrowed areas (Fig. S1). This indicates that the decrease in photosynthetic pigment content is not the main reason for the changes in soluble sugar and reducing sugar contents. In our study, disturbance by rodents significantly increased the contents of total soluble sugar and reducing sugar, while significantly decreased the content of crude fat in assimilating branches of middle-aged $H$. ammodendron (Figs. $2 \mathrm{~b}$, e and f). It may be related to the mechanical damage of photosynthetic tissues by herbivores, changing the status and distribution of carbohydrate in plants (Koch, 1996). In burrowed areas, total soluble sugar and reducing sugar in assimilating branches may be mostly used as osmoregulation substances for middle-aged H. ammodendron. In contrast, total soluble sugar and reducing sugar did not increase, but instead significantly reduced, in assimilating branches of young $H$. ammodendron after being disturbed by rodents (Figs. 2e and f), possibly because young trees lack the well-developed root and stem food stores that are omnipresent in mature plants (Barton, 2016). Hence, after being browsed, young $H$. ammodendron reduced the nutrient content, such as total soluble sugar, reducing sugar, crude fiber and calciumin assimilating branches (Fig. 2) to discourage feeding by rodents. According to the optimal allocation theory, extremely defensive plants produce chemical substances to prevent animal feeding, which might decrease the availability of nutrients for growth and development (Züst et al., 2015; Karasov et al., 2017). However, our study only focused on the levels of nutrients, and any changes in the levels of chemical defense substances needs further study.

Modification of nutrient contents in assimilating branches of $H$. ammodendron due to the disturbance by rodents was only significant in young $H$. ammodendron. Ulappa et al. (2014) reported that browsed plants with a long-term occupancy were more likely to have the greater crude protein content. In our study, there was a significant positive correlation between crude protein and soil $\mathrm{N}$ (Table S1). However, disturbance by rodents only significantly increased the crude protein content in assimilating branches of young $H$. ammodendron (Fig. 2a). The nutrient contents in assimilating branches of mature $H$. ammodendron were virtually unaffected, except for phosphorus contents (Fig. 2). These findings are comparable to the results reported by O'Reilly-Wapstra et al. (2005) and Sotelo et al. (2014). The age at which plants are fed on also affects the optimum defense response (Karasov et al., 2017). Mature plants of the same species might have a stronger capability to defend themselves than young specimens. Thus, the degree of disturbance by rodents seen in burrowed areas of this study was inadequate to have a significant 
impact on the nutrient composition of assimilating branches of mature $H$. ammodendron .

It should be noted that the amount of feeding on $H$. ammodendron by rodents varies with season and burrow density (Liu et al., 2012; Xu et al., 2012). H. ammodendron was much more abundant than any other shrub species and had become the main food source for rodents in the study area during autumn, when most herbaceous plants withered (Liu et al., 2012). We conducted the experiment in summer when the burrow coverage was $18 \%-20 \%$ in this area (Ma et al., 2018). However, with a change in the degree of disturbance, eco-physiological changes in $H$. ammodendron may fluctuate. The results of our study preliminarily examined some of the eco-physiological responses of $H$. ammodendron to the disturbance by rodents in the southern edge of the Gurbantunggut Desert.

\section{Conclusions}

Rodent feeding decreased the photosynthetic pigment contents of $H$. ammodendron but increased the volume of branches and crown to cope with the losses of plant tissue, nutrient and photosynthetic machinery. In response to the losses caused by rodent activity, young $H$. ammodendron tended to reduce the nutrient content of assimilating branches to discourage herbivores from feeding on the branches. The effect of increased soil nutrient on young $H$. ammodendron was more dramatic than those of on middle-aged and mature $H$. ammodendron. Middle-aged $H$. ammodendron tended to invest more energy in defense, increasing their chemical defense substances, such as total soluble sugar and reducing sugar and decreasing the nutrient content of assimilating branches. The response of mature $H$. ammodendron to rodents was the least pronounced of the age groups. Overall, $H$. ammodendron with different age groups has varying degrees of tolerance to the disturbance by rodents. Defense system of $H$. ammodendron increased with the growth of plants and the effect of disturbance by rodents on $H$. ammodendron gradually decreased with age.

\section{Acknowledgements}

The study was funded by the National Natural Science Foundation of China (41561010, 31560177), and the Key Laboratory of Xinjiang Phytomedicine Resource and Utilization and Academic Leader of "3152" in Shihezi University, China. We are grateful to the help from Ms. JIANG Fengjiao, Mr. GE Jinxiong and Mr. LUO Yunfeng.

\section{References}

Allington G R H, Valone T J. 2014. Islands of fertility: A byproduct of grazing? Ecosystems, 17(1): 127-141.

Andino N, Borghi C E. 2018. Occurrence of ctenomys mendocinus in a high-altitude cold desert: Effect on density, biomass, and fitness of sagebrush plants. Arctic, Antarctic, and Alpine Research, 49(1): 53-60.

Bao S D 1999. Methods of Agrochemical Soil Analysis. Beijing: China Agriculture Press, 25-35. (in Chinese)

Barthelemy H, Stark S, Michelsen A, et al. 2018. Urine is an important nitrogen source for plants irrespective of vegetation composition in an Arctic tundra: Insights from a ${ }^{15} \mathrm{~N}$-enriched urea tracer experiment. Journal of Ecology, 106(1): $367-378$.

Barton K E, Koricheva J. 2010. The ontogeny of plant defense and herbivory: characterizing general patterns using meta-analysis. American Naturalist, 175(4): 481-493.

Barton K E. 2016. Low tolerance to simulated herbivory in Hawaiian seedlings despite induced changes in photosynthesis and biomass allocation. Annals of Botany, 117(6): 1053-1062.

Berke S K. 2010. Functional groups of ecosystem engineers: a proposed classification with comments on current issues. Integrative \& Comparative Biology, 50(2): 147-157.

Braak C J F T, Prentice I C. 2004. A theory of gradient analysis. Advances in Ecological Research, 34: 23-28.

Campos-Vargas R, Saltveit M E. 2010. Involvement of putative chemical wound signals in the induction of phenolic metabolism in wounded lettuce. Physiologia Plantarum, 114(1): 73-84.

Christie K S, Ruess R W, Lindberg M S, et al. 2014. Herbivores influence the growth, reproduction, and morphology of a widespread arctic willow. PloS ONE, 9(7): e101716.

Dong B C, Fu T, Luo F L, et al. 2017. Herbivory-induced maternal effects on growth and defense traits in the clonal species Alternanthera philoxeroides. Science of the Total Environment, 605-606: 114-123. 
Ewing S A, Southard R J, Macalady J L, et al. 2007. Soil microbial fingerprints, carbon, and nitrogen in a Mojave Desert creosote-bush ecosystem. Soil Science Society of America Journal, 71(2): 469-475.

Falkenberg J C, Clarke J A. 1998. Microhabitat use of deer mice: Effects of interspecific interaction risks. Journal of Mammalogy, 79(2): 558-565.

Hagenah N, Bennett N C. 2013. Mole rats act as ecosystem engineers within a biodiversity hotspot, the Cape Fynbos. Journal of Zoology, 289(1): 19-26.

Hakes A S, Cronin J T. 2011. Resistance and tolerance to herbivory in Solidago altissima (Asteraceae): Genetic variability, costs, and selection for multiple traits. American Journal of Botany, 98(9): 1446-1455.

He A L, Niu S Q, Zhao Q, et al. 2018. Induced salt tolerance of perennial ryegrass by a novel bacterium strain from the rhizosphere of a desert shrub Haloxylon ammodendron. International Journal of Molecular Sciences, 19(2): 469.

Huang G, Cao Y F, Wang B, et al. 2015. Effects of nitrogen addition on soil microbes and their implications for soil Cemission in the Gurbantunggut Desert, center of the Eurasian Continent. Science of the Total Environment, 515-516: 215-224.

Jiang H P, Wu N, Yang W K. 2007. Effects of Rhombomys opimus on microbial quantity, soil moisture content and soil nutrient content in a desert. Arid Zone Research, 24(2): 187-192. (in Chinese)

Jung S. 2004. Effect of chlorophyll reduction in Arabidopsis thaliana by methyl jasmonate or norflurazon on antioxidant systems. Plant Physiology \& Biochemistry (Paris), 42(3): 225-231.

Kafle D, Wurst S. 2019. Legacy effects of herbivory enhance performance and resistance of progeny plants. Journal of Ecology, 107(1): 58-68.

Kang J J, Zhao W, Ying Z, et al. 2017. Calcium chloride improves photosynthesis and water status in the $\mathrm{C}_{4}$ succulent xerophyte Haloxylon ammodendron under water deficit. Plant Growth Regulation, 82(3): 467-478.

Karasov T, Chae E, Herman J, et al. 2017. Mechanisms to mitigate the tradeoff between growth and defense. Plant and Cell, 29(4): 666-680.

Kindler D S, Robert S. 1970. Nutrients and the reaction of two alfalfa clones to the spotted alfalfa aphid. Journal of Economic Entomology, 63(3): 938-940.

Koch K E. 1996. Carbohydrate-modulated gene expression in plants. Annual Review of Plant Physiology and Plant Molecular Biology, 47(1): 509-540.

Kozlov M V, Zvereva E L. 2012. Delayed local responses of downy birch to damage by leafminers and leafrollers. Oikos, 121(3): 428-434.

Lacey E A, Patton J L, Cameron G N, et al. 2001. Community Ecology of Subterranean Rodents. Chicago: University of Chicago Press, 75-76.

Lepš J, Šmilauer P 2003. Multivariate analysis of ecological data using CANOCO. Cambridge: Cambridge University Press, $60-65$.

Li C J, Li Y, Ma J. 2011. Spatial heterogeneity of soil chemical properties at fine scales induced by Haloxylon ammodendron (Chenopodiaceae) plants in a sandy desert. Ecological Research, 26(2): 385-394.

Li L, Li H X, Zeng H L, et al. 2016. Exogenous jasmonic acid and cytokinin antagonistically regulate rice flag leaf senescence by mediating chlorophyll degradation, membrane deterioration, and senescence-associated genes expression. Journal of Plant Growth Regulation, 35(2): 366-376.

Lichtenthaler H K. 1987. Chlorolshylls and carotenoids: Pigments of photosynthetic biomembranes. Methods in Enzymology, 148: $350-382$.

Liu W, Xu W X, Yang W K, et al. 2012. Food habits of the great gerbil (Rhombomys opimus) in the Southern Gurbantunggut Desert, Xinjiang, China. Pakistan Journal of Zoology, 44(4): 931-936.

Luo Q, Chen Q, Ning H, et al. 2017. Chronosequence-based population structure and natural regeneration of Haloxylon ammodendron plantation in the southern edge of the Gurbantunggut Desert, Northwestern China. Russian Journal of Ecology, 48(4): 364-371.

Ma T, Zheng J H, Wen A M, et al. 2018. Group coverage of burrow entrances and distribution characteristics of desert forest-dwelling Rhombomys opimus based on unmanned aerial vehicle (UAV) low-altitude remote sensing: A case study at the southern margin of the Gurbantunggut Desert in Xinjiang. Acta Ecologica Sinica, 38(3): 953-963. (in Chinese)

Mabry T J, Hunziker J H, Difeo D R J. 1977. Creosote bush: Biology and chemistry of Larrea in new world deserts. Desert Plants, 31(5): 399.

Mao L Z, Lu H F, Wang Q, et al. 2007. Comparative photosynthesis characteristics of Calycanthus chinensis and Chimonanthus praecox. Photosynthetica, 45(4): 601-605.

Mares M A, Ojeda R A, Borghi C E, et al. 1997. How desert rodents overcome halophytic plant defenses. Bioscience, 47(10): 699-704. 
Munns R, Tester M. 2008. Mechanisms of salinity tolerance. Annual Review of Plant Biology, 59(1): 651-681.

Nabity P D, Zavala J A, DeLucia E H. 2013. Herbivore induction of jasmonic acid and chemical defences reduce photosynthesis in Nicotiana attenuata. Journal of Experimental Botany, 64(2): 685-694.

Nelson N. 1944. A photometric adaptation of the Somogyi method for determination of glucose. Journal of Biological Chemistry, 153: 375-380.

Ngatia L W, Turner B L, Njoka J T, et al. 2015. The effects of herbivory and nutrients on plant biomass and carbon storage in vertisols of an East African savanna. Agriculture Ecosystems \& Environment, 208: 55-63.

O'Reilly-Wapstra J M, Potts B M, McArthur C, et al. 2005. Inheritance of resistance to mammalian herbivores and of plant defensive chemistry in a Eucalyptus species. Journal of Chemical Ecology, 31(3): 519-537.

Roe J H. 1955. The determination of sugar in blood and spinal fluidwith anthrone reagent. Journal of Biological Chemistry, 212(1): 335-343.

Rogovin K, Randall J A, Kolosova I, et al. 2003. Social correlates of stress in adult males of the great gerbil, Rhombomys opimus, in years of high and low population densities. Hormones \& Behavior, 43(1): 132-139.

Schweissing F C, Wilde G. 1979. Temperature and plant nutrient effects on resistance of seedling sorghum to the greenhug. Journal of Economic Entomology, 72(1): 20-23.

Shimizu-Sato S, Tanaka M, Mori H. 2009. Auxin-cytokinin interactions in the control of shoot branching. Plant Molecular Biology, 69(4): 429-435.

Siemens D H, Garner S H, Mitchell-Olds T, et al. 2002. Cost of defense in the context of plant competition: Brassica rapa may grow and defend. Ecology, 83(2): 505-517.

Sitters J, te Beest M, Cherif M, et al. 2017. Interactive effects between reindeer and habitat fertility drive soil nutrient availabilities in arctic tundra. Ecosystems, 20(7): 1266-1277.

Sivritepe N, Kumral N A, Erturk U, et al. 2009. Responses of grapevines to two-spotted spider mite mediated biotic stress. Journal of Biological Sciences, 9(4): 311-318.

Somogyi M. 1952. Note on sugar determination. Journal of Biological Chemistry, 195(1): 19-23.

Sotelo P, Pérez E, Najar-Rodriguez A, et al. 2014. Brassica plant responses to mild herbivore stress elicited by two specialist insects from different feeding guilds. Journal of Chemical Ecology, 40(2): 136-149.

Steingraeber D A, Waller D M. 1986. Non-stationarity of tree branching patterns and bifurcation ratios. Series B, Biological Sciences, 228(1251): 187-194.

Tchabovsky A V, Krasnov B, Khokhlova I S, et al. 2001. The effect of vegetation cover on vigilance and foraging tactics in the fat sand rat Psammomys obesus. Journal of Ethology, 19(2): 105-113.

Ulappa A C, Kelsey R G, Frye G G, et al. 2014. Plant protein and secondary metabolites influence diet selection in a mammalian specialist herbivore. Journal of Mammalogy, 95(4): 834-842.

Whitford W G, Kay F R. 1999. Biopedturbation by mammals in deserts: a review. Journal of Arid Environments, 41(2): 203-230.

Whitney G G. 1976. The bifurcation ratio as an indicator of adaptive strategy in woody plant species. Bulletin of the Torrey Botanical Club, 103(2): 67-72.

$\mathrm{Xu}$ M H, Liu T, Jiang L. 2012. Study on the harm characteristics of rodents to Haloxylon ammodendrom and its control for ecological threshold value in the south of Gurbantonggut Desert. Journal of Arid Land Resources and Environment, 26(6): 126-133. (in Chinese)

Xu W X, Liu W, Yang W K, et al. 2012. Rhombomys opimus contribution to the "fertile island" effect of tamarisk mounds in Junggar Basin. Ecological Research, 27(4): 775-781.

Zhang K, Su Y Z, Liu T N, et al. 2016. Leaf C:N:P stoichiometrical and morphological traits of Haloxylon ammodendron over plantation age sequences in an oasis-desert ecotone in North China. Ecological Research, 31(3): 449-457.

Zhang S L, Kou M J, Bing J C, et al. 2001. Investigation of forest rodents fauna in Gansu province. Forest Pest and Disease, 6 : 26-28. (in Chinese)

Zhang S L, Chen Y W, Ma J M, et al. 2009. Effect of the damage and feeding of Rhombimys opimus on the growth of Haloxylon ammodendron. Forest Pest \& Disease, 28(1): 7-9. (in Chinese)

Züst T, Rasmann S, Agrawal A A. 2015. Growth-defense tradeoffs for two major anti-herbivore traits of the common milkweed Asclepias syriaca. Oikos, 124(10): 1404-1415. 


\section{Appendix}
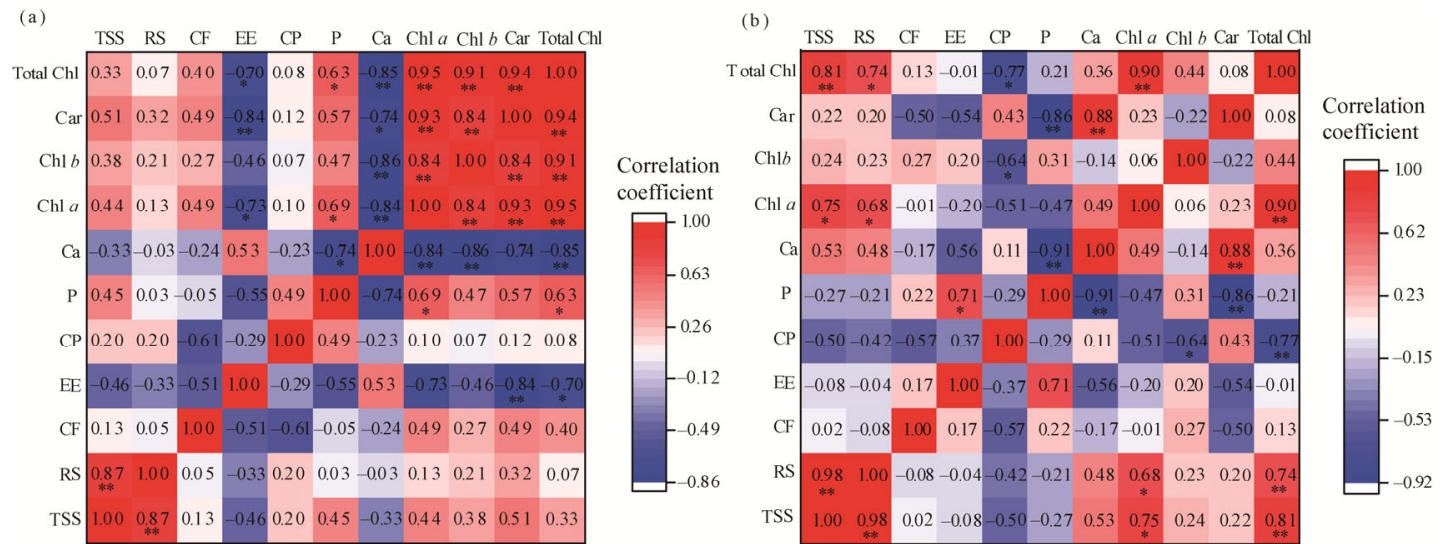

Fig. S1 Pearson's correlation coefficient among physiological indices of assimilating branches of $H$. ammodendron in burrowed and control areas. $*$ and $* *$ indicate significant differences between these indices at $P<0.05$ and $P<0.01$ levels, respectively. TSS, total soluble sugar; RS, reducing sugar; CF, crude fiber; EE, crude fat; $\mathrm{CP}$, crude protein; $\mathrm{P}$, phosphorous; $\mathrm{Ca}$, calcium; Cha, Chlorophyll $a$; Chb, Chlorophyll $b$; Car, carotenoid; Total Chl, total chlorophyll.

Table S1 Correlation of nutrient between topsoil and assimilating branches of $H$. ammodendron in burrowed area

\begin{tabular}{cccccccc}
\hline & SOM & TN & TP & TK & AN & AP & AK \\
\hline TSS & 0.58 & -0.32 & -0.55 & -0.21 & -0.35 & 0.50 & -0.46 \\
RS & 0.55 & -0.38 & -0.52 & -0.17 & -0.40 & 0.35 & -0.29 \\
CF & 0.06 & -0.47 & 0.03 & 0.26 & -0.35 & -0.06 & 0.34 \\
EE & -0.38 & $-0.82^{* *}$ & 0.12 & -0.30 & $-0.79^{*}$ & -0.43 & -0.31 \\
CP & -0.11 & $0.62^{*}$ & $0.70^{*}$ & 0.15 & $0.75^{*}$ & 0.18 & $0.78^{*}$ \\
P & 0.03 & 0.61 & 0.54 & 0.11 & $0.78^{*}$ & $0.71^{*}$ & 0.40 \\
Ca & 0.14 & $-0.74^{*}$ & -0.54 & -0.43 & $-0.88^{* *}$ & -0.31 & $-0.82^{* *}$ \\
\hline
\end{tabular}

Note: TSS, total soluble sugar; RS, reducing sugar; CF, crude fiber; EE, crude fat; CP, crude protein; P, phosphorous; Ca, calcium; SOM, soil organic matter; TN, total nitrogen; TP, total phosphorus; TK, total potassium; AN, available nitrogen; AP, available phosphorus; AK, available potassium. ${ }^{*}$ and ${ }^{* *}$ indicate significant correlations at $P<0.05$ and $P<0.01$ levels, respectively. 